\title{
The Clinical Relevance of Gastroesophageal Reflux Disease and Laryngopharyngeal Reflux in Clinical Practice
}

\author{
Aragona Salvatore Emanuele, \\ Mereghetti Giada and Giorgio Ciprandi \\ Additional information is available at the end of the chapter \\ http://dx.doi.org/10.5772/intechopen.78357
}

\begin{abstract}
Gastric reflux may be considered a para-physiological event that may occur up to 50 times a day. It usually happens when gas (less commonly liquids) flow back from stomach into esophagus. However, when defense mechanisms leave, disease may progress. If the esophagus is the trigger, gastroesophageal reflux disease (GERD) emerges. The prevalence of GERD in the primary care setting seems to be even more evident when one considers that, in the United States, 4.6 million office encounters annually are primarily for GERD, whereas 9.1 million encounters include GERD in the top 3 diagnoses for the encounter. GERD constitutes also the most frequently first-listed gastrointestinal diagnosis in ambulatory care visits. In addition, the extraesophageal manifestations of reflux, including LPR, asthma, and chronic cough, have been estimated to cost $\$ 5438$ per patient in direct medical expenses in the first year after presentation and $\$ 13,700$ for 5 years. Presently, the newest alginate compounds renowned the interest in this attracting and stimulating area. In this regard, a new medical device (Marial $\left.{ }^{\circledR}\right)$, unique still now possessing the indication for both GERD and LPR, has been recently launched in the Italian market, two large surveys were conducted in Italy: RELIEF, involving 86 otolaryngologists, and EMERGE, involving 56 gastroenterologists. The aims of these surveys were: (1) to define clinical characteristics, including previous treatment, of the patients referred to consultation; (2) to evaluate the reliability of RFS, GIS, and RSI questionnaires in real-world settings, such as specialist office; and (3) to investigate the patients' perception of efficacy of the prescribed therapy, based on the best practice and considering also the new medical device Comparing the patients' perception of treatment efficacy, reduction in RSI values for each single symptom before and after a 4 week-treatment with Marial ${ }^{\circledR}$ alone or with PPI in add-on in EMERGE and RELIEF patients are reported. Marial ${ }^{\circledR}$ alone treatment induced a statistically significant higher reduction in each single symptom in RELIEF patients than in EMERGE patients, with the exception of heartburn, chest pain, indigestion, or stomach
\end{abstract}


acid coming up. Similar results were obtained evaluating the reduction in RSI values in patients treated with PPI in add-on that was able to determine a higher statistically significant decrease in RELIEF than in EMERGE patients in each single symptom, with the exception of heartburn, chest pain, indigestion, or stomach acid coming up.

Keywords: regenerative medicine, GERD, LPR

\section{Background}

Actually, gastric reflux may be considered a para-physiological event that may occur up to 50 times a day. It usually happens when gas (less commonly liquids) flow back from stomach into esophagus. However, when defense mechanisms leave, disease may progress. If the esophagus is the trigger, gastroesophageal reflux disease (GERD) emerges. On the other hand, laryngopharyngeal reflux (LPR) is considered an extraesophageal manifestation of the GERD. Both GERD and its extraesophageal manifestation are very common in clinical practice. Both disorders have a relevant burden for the society: many pharmaco-economic studies were conducted in the United States. In population-based studies, $19.8 \%$ of North Americans complain of GERD symptoms, including heartburn and regurgitation, at least weekly [1]. Likewise, in the late 1990s, GERD accounted for $\$ 9.3$ to $\$ 12.1$ billion in direct annual healthcare costs in the United States, higher than any other digestive disease. Consequently, acidsuppressive agents are still the leading pharmaceutical expenditure in the United States.

The prevalence of GERD in the primary care setting seems to be even more evident when one considers that, in the United States, 4.6 million office encounters annually are primarily for GERD, whereas 9.1 million encounters include GERD in the top 3 diagnoses for the encounter. GERD constitutes also the most frequently first-listed gastrointestinal diagnosis in ambulatory care visits $[2,3]$. In addition, the extraesophageal manifestations of reflux, including LPR, asthma, and chronic cough, have been estimated to cost $\$ 5438$ per patient in direct medical expenses in the first year after presentation and $\$ 13,700$ for 5 years. Estimates of the economic burden of extraesophageal reflux have shown that expenditures for extraesophageal manifestations of reflux could surpass $\$ 50$ billion, $86 \%$ of which could be attributable to pharmaceutical costs $[2,3]$. In addition, the National Health Care Survey carried out by the Center for Disease Control and Prevention has reported that the main complaint for primary care patient visits was cough in $6.1 \%$, throat symptoms in $4 \%$, and asthma in $2.8 \%$ [4]. Within these visits for cough, asthma, and throat symptoms are contained the hidden prevalence of extraesophageal manifestations of GERD, which to date have not been adequately addressed from a medical or surgical perspective due to their perceived obscurity. Therefore, the gastric reflux, globally understood, represents a very important medical issue that deserves adequate attention in daily practice.

From a pathophysiological point of view, gastric reflux includes different mechanisms, such as the provocation and perception of reflux. The transient lower esophageal sphincter relaxations (TLESR), hiatus hernia, acid pocket, visceral hypersensitivity, and obesity represent important causes of gastric reflux. Impaired esophageal, and extraesophageal, mucosal 
integrity, poor esophageal clearance, and delayed gastric emptying could be associated with GERD development. In addition, another pathogenic factor is a neural reflex sustained by acid exposure: the so-called Reflex-Reflux [5].

Distinguishing whether cough, LPR, and asthma may be sustained by GERD remains difficult challenging for both the primary care physician and the specialist. The distinction between them is clinically relevant as treatment of GERD with the intent of improving or curing extraesophageal manifestation could be ineffective. To review the current literature on extraesophageal manifestations of reflux should assist in clinical decision making.

The Montreal Classification gave the most recent consensus definition of GERD. This document defines GERD as heartburn symptoms or complications resulting from the reflux of gastric contents into the esophagus, up to the oral cavity, and lungs [5]. GERD is again classified into two subgroups. The first subgroup is represented by GERD with heartburn symptoms but without endoscopic evidence of mucosal erosions (the so called Non-Erosive Reflux Disease or NERD). The second sub-group is GERD with heartburn symptoms accompanied by objective evidence of erosions, ulcers, and inflammation (the so called Erosive Reflux Disease or ERD) [6].

It has to be considered that functional heartburn might fall under endoscopic negative disease. However, it is important to note that it is a distinct entity from NERD. NERD is usually defined as typical reflux symptoms without evidence of reflux disease in endoscopy but abnormal acid exposure on the impedance-pH monitoring and is responsive to proton pump inhibitors PPI $[7,8]$. Functional heartburn on the other hand, as defined by Rome IV classification, is a retrosternal burning discomfort or pain refractory to anti-secretory therapy without presence of GERD, histopathologic abnormality, motility disorder or structural abnormality for at least 3 months with symptoms onset at least 6 months prior to the diagnosis [5].

As just defined, stomach content may also reflux outside of the esophagus into respiratory organs, such as extraesophageal reflux, including LPR. LPR is most commonly manifested as laryngeal symptoms such as coughing, hoarseness, dysphagia, globus, and sore throat, but there can be signs also of nose, sinus, ear, and eye involvement [9]. Epidemiological studies have shown that the prevalence of this LPR may be extremely high, that it has certain characteristics of an outbreak and that it is one of the most common causes of patient visits to their family medicine physicians, but also to otolaryngologists, gastroenterologists, pediatricians, pulmonologists, allergists, and psychiatrists [1, 10-13]. Today it has been proven that gastroesophageal reflux is not the only cause of LPR. LPR is a multifactorial syndrome with a vast clinical representation, during the disease and with complications, so it requires and deserves a multidisciplinary approach. Based on newly discovered findings about the specific pathogenesis of the disease, LPR may be considered a new clinical entity [11-13]. As once pointed out, GERD is caused by the lower esophageal sphincter dysfunction and the dysfunction of the stomach emptying mechanism. Esophageal mucosa has protective mechanisms against aggressive factors of the stomach content (mucosal barrier) and it remains intact when a physiological reflux occurs, which normally happens at night. However, laryngeal and pharyngeal mucosa do not possess the esophageal protective mechanisms, so acid and peptic activity of the stomach content quickly leads to mucosal lesions. Notably, laryngopharyngeal reflux occurs most commonly during the day as a result of the upper esophageal 
sphincter dysfunction. This aspect is intriguing as typical GERD symptoms usually occur in supine position and overnight. However, acidity of the stomach content is not the only cause of LPR. Pepsin with its proteolytic effects can be the determining factor. Other possible etiological factors are pancreatic proteolytic enzymes, bile salts, and bacteria [1, 13, 14]. Extraesophageal manifestations of stomach content reflux have only recently started being seen as important based on the assumption of their important role in causing respiratory tract diseases. In clinical practice, LPR is mostly not recognized because it may be a "silent reflux" and diagnostic and therapeutic protocols are still inadequate, so proper treatment is usually delayed. Laryngeal symptoms are the most common, so patients are managed by otolaryngologists. Indeed, otolaryngologists have developed the diagnostic Reflux Symptom Index (RSI) questionnaire based on the importance of certain disease symptoms and the Reflux Finding Score (RFS) based on frequency of pathological changes determined by laryngoscopy [15]. On the other hand, considering the high prevalence of the disease and uncharacteristic clinical image, most patients report to their family medicine physicians [14-17]. For family medicine physicians LPR represents an important medical problem and a challenge in fast diagnostics, proper treatment, and proper selection of patients who require additional multidisciplinary diagnostic procedures. Knowledge of pathogenic pathway of the disease and its clinical manifestations can help physicians in creating an adequate program for prevention, early diagnosis, and adequate therapy for LPR. In particular, it has to be considered that untreated LPR can be one of the etiological causes of laryngeal cancer. The development of the disease can be benign or malignant and life threatening, and all of its forms can considerably affect life quality in patients. Laryngeal pathological changes could be discovered with laryngoscopy, and some even with detailed esophagogastroscopy. These changes may include: edema, hyperemia, or erythema of the vocal chords and laryngeal edges, ventricular obliteration, granulation, presence of dense endolaryngeal secretion, and hypertrophy of the posterior commissure [10, 15]. As consequence, an appropriate diagnosis of LPR represents a challenge for general practitioner and specialists. A large number of clinical studies confirmed low specificity and sensitivity of diagnostic tests such as laryngoscopy, esophagogastroscopy, proximal $\mathrm{pH}$ monitoring (hypopharyngeal and oropharyngeal). Evaluation of symptoms using the Reflux Symptom Index is considered to be the basic diagnostic procedure. A newer method of measuring salivary pepsin (Pep-test) can confirm LPR diagnosis because its sensitivity and specificity is $87 \%$ [13]. In this regard, it has to be noted that pepsinogen is produced only in the stomach, so pepsin may be envisaged as a specific biomarker for gastric reflux. The Pep-test is a fast and non-invasive method and could have a wide variety of uses in primary health care.

LPR therapy is complex and requires also modification of the patient's lifestyle and habits. Body weight reduction and physical activity, quitting cigarettes and alcohol use are one of the first steps in lowering the intensity of symptoms in patients [17]. Nutritional interventions with correct food choices and bowel movement regulation lead to lowering dyspeptic complains, but also lower the number of reflux episodes. Emptying of the bowels causes lower intra-abdominal pressure, which leads to lower possibility of stomach content reflux into the esophagus, larynx and pharynx. Obesity, or more precisely high BMI, so including overweight, is an independent factor in stomach reflux occurrence because of its specific effect mechanism on the gastroesophageal juncture [17]. LPR treatment and management is 
supposed to reduce the acidity or stomach contents and neutralize acid-peptic activity in larynx, pharynx and esophagus. High dosages of PPI (proton pump inhibitors) have shown the best effects in reducing reflux in the course of $24 \mathrm{~h}$. Alkaline water and alginates show a positive additional effect in lowering acid-peptic activity in the larynx and pharynx. Patients are supposed to have long-term treatment during the course of 6 months because of high sensitivity of the mucosal membrane in the stomach and pharynx. Difficult cases with a proven hiatal hernia can be considered for surgical treatment as well [6].

Therefore, acid suppression is the mainstay of therapy for gastric reflux, and PPIs are the most effective drug in this approach $[18,19]$. Although PPIs are the treatment of choice for GERD, still approximately one-third of patients with GERD fail to respond symptomatically to a standard dose PPI, either partially or completely [20]. Actually, NERD accounts for $60-70 \%$ of GERD patients and is considered the most common presentation of GERD. However, only approximately $30-40 \%$ of NERD patients respond to a standard dose of PPIs, much lower than that in erosive esophagitis, and the low response rate to PPIs in NERD patients is the main contributor to the high portion of PPI failure phenomenon in GERD, and also LPR, management [21]. The mechanisms of failure of PPI therapy are complex and multifactorial [20, 22-24]. Consequently, other medications should be considered and used. In this context, alginates and histamine type-2 receptor antagonists (H2RAs) may provide additional benefit for symptom relief in patients with persistent symptoms despite PPI therapy and can be considered as addon therapy for patients who fail with a PPI. However, because of the concern about tolerance, H2RA is suggested to be taken on demand or intermittently. PPI-refractory GERD (and LPR), defined as persistent reflux symptoms not responding to a double dose of a PPI therapy during a treatment period of at least 12 weeks, is an important issue in clinical practice and poses a great challenge for general practitioners, internists, gastroenterologists, and otolaryngologists [20]. Compliance with therapy should be verified first by the physician, and the presence of functional gastrointestinal disorders, psychological distress, functional heartburn or other esophagitis not related to reflux should also be carefully evaluated in these patients.

On the basis of these concepts, alginate may be considered a fruitful and relevant option in many patients with reflux disease. In particular, the knowledge about the utility of alginates derives from an interesting research area investigating the pathogenic role of the so-called "acid pocket." The acid pocket is a short zone of unbuffered highly acidic gastric juice that accumulates in the proximal stomach after meals. Serving as the source of acid reflux, the acid pocket increases the propensity for acid reflux by all conventional mechanisms, such as TLESR and hiatus hernia, and has been considered as an important cause of GERD $[25,26]$. Alginate is an anionic polysaccharide occurring naturally in brown algae and has a unique property in the treatment of gastric reflux by eliminating the acid pocket. Alginate-antacid formulation can reduce postprandial symptoms by neutralizing the acidity of gastric contents. In addition to neutralizing the gastric acidity, more importantly, alginate and bicarbonate, usually contained in an alginate-based formulation, form a foamy gel that is like a raft floating on the surface of gastric contents after interacting with gastric acid, and this barrier-like gel displaces the acid pocket from the esophageal-gastric junction and protects both the esophageal and the upper respiratory mucosa from the acid and non-acid reflux by gel coating [27-30]. Like an antacid, an alginate-based formulation demonstrates 
an immediate onset of effect within $1 \mathrm{~h}$ of administration, which is faster than a PPI and H2RA [31]. Compared with antacids, an alginate-based formulation is more effective than an antacid in controlling postprandial esophageal acid exposure and quickly relieving reflux symptoms, including heartburn, regurgitation, vomiting and belching, with longer duration [32-34]. Alginate-based formulations are also non-inferior to omeprazole in achieving a heartburn-free period in moderate episodic heartburn [35]. Therefore, alginate has the special properties of protection of the esophageal and upper respiratory mucosa from acid and nonacid reflux and displacement of acid pocket away from the esophagus, all of which make alginate an attractive agent in the management of refractory reflux symptoms with a cause other than by acid, such as NERD [36]. Compared with placebo, an alginate-antacid formulation demonstrated superior relief of reflux symptoms including heartburn and regurgitation in both patients with NERD and erosive esophagitis in a double-blind randomized controlled trial [37]. In another double-blind randomized clinical trial comparing the efficacy of alginate to omeprazole in patients with NERD, alginate demonstrated non-inferiority to omeprazole and was as effective as omeprazole for symptomatic relief [38]. Furthermore, adding alginate to a PPI can significantly relieve heartburn compared to using a PPI alone in patients with NERD, suggesting an additional benefit of alginate as add-on therapy in the management of refractory symptoms [39].

Interestingly, in a meta-analysis study, six of nine randomized trials found no difference between the PPI and placebo groups for LPR, whereas three trials exhibited statistically significant results [1]. In a systemic review, three of four randomized controlled studies revealed that prokinetic agents significantly reduced LPR symptoms, but there were too many study limitations to draw firm conclusions [40]. In a small randomized controlled study, a liquid alginate suspension could achieve significant improvement in the symptom scores and clinical findings of LPR [41].

Therefore, on the basis of this discussed background, the management of suspected LPR is intriguing as it is very difficult, if even possible, to make a definitive diagnosis with the tools currently available [42]. If there is no doubt that many patients do have LPR symptoms, the probability of suspecting LPR, especially when typical reflux symptoms are lacking and PPIs do not improve symptoms, are low, mainly in non-specialist setting. LPR management is responsible of high economic burden mainly related to the prescription of PPIs, which may be, in most cases, not justified $[3,43,44]$. Therefore, in patients, initially visited by GP, who do not respond to a 2 to 3 -month course of double dose PPI therapy, the role of the otolaryngologist is to document the presence of signs and symptoms suggestive of LPR with appropriate (i.e., validated and reproducible) investigations, namely fiber-endoscopy, and validated questionnaire (for example RSI and RFS). If LPR is documented, it is reasonable empirically testing these patients on PPIs to check for reflux control may be useful to select PPI-responder patients. If PPI are not adequate to control symptoms an add-on treatment should be prescribed. On the basis of the above-mentioned concepts, alginate could be a firstchoice option. As a matter of the fact, as there is no specific and focused medication able to irreversibly inactivate pepsin and block acid production, other compounds have place in LPR management, including medical devices with barrier effect. In the current scenario, an effective supportive strategy may be constituted by compounds able to strengthen the epithelial 
barrier providing protection from acid and pepsin and promoting mucosal healing. In other words, an old concept could be revised for LPR therapy: the "cytoprotection" of mucosal tissues [45]. Mucosal cytoprotection was an ideal target of two main drug classes: prostaglandins and sucralfate. Many clinical trials supported this theory that met favorable impression some decades ago [46-49].

Presently, the newest alginate compounds renowned the interest in this attracting and stimulating area. In this regard, a new medical device (Marial@), unique still now possessing the indication for both GERD and LPR, has been recently launched in the Italian market. It is an innovative gel compound, containing magnesium alginate and E-Gastryal ${ }^{\circledR}$ (hyaluronic acid, hydrolyzed keratin, Tara gum, and Xantana gum). E-Gastryal ${ }^{\circledR}$ is a complex of phyto-polymers, Tara and Xantana gums, that are natural polysaccharides with high molecular weight and partially hydrosoluble, and able to provide viscosity to the solution and to generate a support frame where keratin peptide chains and hyaluronic acid anchor. Hyaluronic acid (HA) is a biopolymer with medium molecular weight characterized by optimal hygroscopic and hydrodynamic features. The chemical-physical properties of the polymeric complex confer mucoadhesiveness to E-Gastryal ${ }^{\circledR}$ so increasing the contact surface and the residence time on the mucous membranes of larynx, pharynx, and esophagus. In this context, hyaluronic acid is extremely bioavailable and able to carries out its activity aimed to induce repairing and regenerating the damaged epithelium. HA, by its hydrophilic essence, realizes a favorable milieu for cellular migration; in addition, HA, having a scavenger activity of free radicals, exerts a protective role towards oxide damage and proteolytic enzymes, such as pepsin.

Hydrolyzed keratin, an indigestible substance, increases the solidity and the resistance of E-Gastryal ${ }^{\circledR}$, enhancing the barrier effect. Really, keratin abounds with cysteine, amino acid sulfide, that forms disulfide bridges extremely firm and able to link the amino acid chains, making an helical structure characterized by difficult dissolution and resistant to attack of acid and pepsin. The alginate has the peculiar property of a boating raft at the acid pocket and selectively inhibits pepsin by mannuronic acid, highly contained in the specific alginate [50]. In particular, this medical device contains magnesium alginate with high ratio mannuronic acid/glucuronic acid and with raised viscosity shaping a stable and compact raft.

For these reasons, two large surveys were conducted in Italy, involving both otolaryngologists and gastroenterologists. The aims were to define the patients' characteristics, including the clinical features, the assessment of treatments, and new therapeutic approaches in view of the new medical device Marial®.

\section{Clinical experience}

As pointed out, the gastroesophageal reflux is considered a normal physiological process that usually happens after eating in healthy infants, children, young people and adults. In contrast, gastroesophageal reflux disease occurs when the effect of GER leads to symptoms severe enough to merit medical treatment. In clinical practice, it is difficult to differentiate 
between GER and GERD, and the terms are used interchangeably by health professionals and families alike. There is no simple, reliable and accurate diagnostic test to confirm whether the condition is GER or GERD, and this in turn affects research and clinical decisions [50-52]. Furthermore, the term GERD covers a number of specific conditions that have different effects and present in different ways. This makes it difficult to identify the person who genuinely has GERD, and to estimate the real prevalence and burden of the problem. Nevertheless, regardless of the definition used, GERD affects many subjects, who commonly seek advice from primary, secondary or tertiary care. As a result, it constitutes a major health burden for the Health Service. Moreover, if gastric refluxate moves more proximally into the laryngopharynx, it is defined laryngopharyngeal reflux (LPR). LPR should be considered as part of extraesophageal reflux (EER), reflux involving structures other than, or in addition to, the esophagus, and airway reflux involving proximal gastric reflux into the airways. LPR contributes to several otorhinolaryngologic symptoms and inflammatory disorders, and probably also to neoplastic diseases of the laryngopharynx, and seems to be also as common in children and infants as adults.

From a diagnostic point of view, GERD and LPR diagnosis may be performed on a clinical ground as there is no gold-standard diagnostic tool. In this regard, some questionnaires may very fruitful in clinical practice: Reflux Finding Score (RFS) based on signs viewed by laryngoscopy, Reflux Score Index (RSI) based on reflux symptoms, and GERD Impact Scale (GIS) based on frequency of symptoms, as reported in the tables (Tables 1-3).

\begin{tabular}{lllll}
\hline & $\begin{array}{l}\text { None of } \\
\text { the time }\end{array}$ & $\begin{array}{l}\text { A little of } \\
\text { the time }\end{array}$ & $\begin{array}{l}\text { Some of } \\
\text { the time }\end{array}$ & $\begin{array}{l}\text { All of the } \\
\text { time }\end{array}$ \\
1 & 2 & 3 & 4 \\
\hline
\end{tabular}

In the past week

1. How often have you had the following symptoms

a. Pain in chest/behind the breastbone?

b. Burning sensation in your chest or behind the breastbone?

c. Regurgitation or acid taste in your mouth?

d. Pain or burning in upper stomach?

e. Sore throat or hoarseness that is related to your heartburn or acid reflux?

2. How often have you had difficulty in getting a good night's sleep because of your symptoms?

3. How often have your symptoms prevented you from eating or drinking any of the foods you like?

4. How often have your symptoms kept you from being fully productive in your job or daily activities?

5. How often do you take additional medication other than what the physician told you to take (such as Gaviscon, Maalox)?

Table 1. GERD Impact Scale (GIS). 


\begin{tabular}{|c|c|c|c|c|c|c|}
\hline \multirow{2}{*}{$\begin{array}{l}\text { Within the last month, how did the following problems affect you? } \\
\text { 1. Hoarseness or a problem with your voice }\end{array}$} & \multicolumn{6}{|c|}{$\begin{array}{l}0=\text { no problem } \\
5=\text { severe problem }\end{array}$} \\
\hline & 0 & 1 & 2 & 3 & 4 & 5 \\
\hline 2. Clearing your throat & 0 & 1 & 2 & 3 & 4 & 5 \\
\hline 3. Excess throat mucus or postnasal drip & 0 & 1 & 2 & 3 & 4 & 5 \\
\hline 4. Difficulty swallowing food, liquids, or pills & 0 & 1 & 2 & 3 & 4 & 5 \\
\hline 5. Coughing after you ate or after lying down & 0 & 1 & 2 & 3 & 4 & 5 \\
\hline 6. Breathing difficulties or choking episodes & 0 & 1 & 2 & 3 & 4 & 5 \\
\hline 7. Troublesome or annoying cough & 0 & 1 & 2 & 3 & 4 & 5 \\
\hline 8. Sensation of something sticking in your throat or a lump in your throat & 0 & 1 & 2 & 3 & 4 & 5 \\
\hline 9. Heartburn, chest pain, indigestion, or stomach acid coming up & 0 & 1 & 2 & 3 & 4 & 5 \\
\hline RSI $>13$ = Abnormal & \multicolumn{6}{|c|}{ Total } \\
\hline
\end{tabular}

Abbreviation: RSI, reflux symptom index.

Table 2. Reflux Symptom Index.

From a management point of view, the guidelines suggest to give advice about GER and reassure patients and caregivers. Patients with dyspepsia with mild-moderate symptoms and without severe complications, such as bleeding, painful complaints, vomiting, could be treated with empirical full-dose PPI therapy for 4 weeks. Patients with GERD could be treated with a full-dose PPI for 4 or 8 weeks. If symptoms recur after initial treatment, a PPI could be offered at the lowest dose possible to control symptoms. In addition, it is recommended to encourage people who need long-term management of dyspepsia symptoms to reduce their use of prescribed medication stepwise: by using the effective lowest dose, by trying 'as-needed' use when appropriate, and by returning to self-treatment with antacid and/or alginate therapy (unless there is an underlying condition or co-medication that needs continuing treatment). It is also necessary to advise people that it may be appropriate for them to return to self-treatment with antacid and/or alginate therapy (either prescribed or purchased over-the-counter and taken as needed). Finally, it is important to avoid long-term, frequent dose and continuous antacid therapy (it only relieves symptoms in the short term rather than preventing them).

On the basis of these considerations, proposed by guidelines, alginates may be considered as a valid and reasonable therapeutic option. A new medical device (Marial ${ }^{\circledR}$ ), unique still now possessing the indication for both GERD and LPR, has been recently launched in the Italian market [53]. It is an innovative gel compound, containing magnesium alginate and E-Gastryal ${ }^{\circledR}$. E-Gastryal $\AA$ is a complex of phyto-polymers, keratin, Tara and Xantana gums, that are natural polysaccharides with high molecular weight and partially hydrosoluble, and able to provide viscosity to the solution and to generate a support frame where keratin peptide chains and hyaluronic acid anchor. Hyaluronic acid (HA) is a biopolymer with medium molecular weight characterized by optimal hygroscopic and hydrodynamic features. The chemical-physical properties of the polymeric complex confer mucoadhesiveness to E-Gastryal ${ }^{\circledR}$ so increasing the contact surface and the residence time on the mucous membranes of larynx, pharynx, and esophagus. 


\begin{tabular}{|c|c|}
\hline \multirow[t]{2}{*}{ Subglottic edema } & $0=a b s e n t$ \\
\hline & $2=$ present \\
\hline \multirow[t]{2}{*}{ Ventricular edema } & $2=$ partial \\
\hline & $4=$ complete \\
\hline \multirow[t]{2}{*}{ Erythema/hyperemia } & $2=$ arytenoids only \\
\hline & $4=$ diffuse \\
\hline \multirow[t]{4}{*}{ Vocal-fold edema } & $1=$ mild \\
\hline & $2=$ moderate \\
\hline & $3=$ severe \\
\hline & 4 = polypoid \\
\hline \multirow[t]{4}{*}{ Diffuse laryngeal edema } & $1=$ mild \\
\hline & $2=$ moderate \\
\hline & $3=$ severe \\
\hline & $4=$ obstructing \\
\hline \multirow[t]{4}{*}{ Posterior commissure hypertrophy } & $1=$ mild \\
\hline & $2=$ moderate \\
\hline & $3=$ severe \\
\hline & 4 = obstructing \\
\hline \multirow[t]{2}{*}{ Granuloma/granulation tissue } & $0=a b s e n t$ \\
\hline & $2=$ present \\
\hline \multirow[t]{2}{*}{ Thicken laryngeal mucus } & $0=a b s e n t$ \\
\hline & $2=$ present \\
\hline
\end{tabular}

RFS, Reflux Finding Score. RFS > 7 suspect of laryngopharyngeal reflux (LPR).

Table 3. Reflux Finding Score.

Therefore, two large surveys were conducted in Italy: RELIEF, involving 86 otolaryngologists, and EMERGE, involving 56 gastroenterologists [54-56]. The aims of these surveys were: (1) to define clinical characteristics, including previous treatment, of the patients referred to consultation; (2) to evaluate the reliability of RFS, GIS, and RSI questionnaires in real-world settings, such as specialist office; and (3) to investigate the patients' perception of efficacy of the prescribed therapy, based on the best practice and considering also the new medical device.

The outcomes of these surveys are here presented and discussed. Now, we would draw the conclusive remarks from a pragmatic point of view. So, we compared the most relevant outcomes obtained by the two surveys.

First, we compared RSI questionnaires: at baseline, RSI questionnaire was filled by 1934 RELIEF patients and by 789 EMERGE patients. Globally, 594 (75.3\%) EMERGE patients and 1250 
(64.6\%) RELIEF patients had positive RSI score. RSI values for each single symptom are analytically reported in Figure 1. The symptoms with the highest score were: heartburning, sensation of something sticking in the throat or a lump in the throat, and clearing throat, whereas breathing difficulties or choking episodes had the lowest score. The total score was 16 [14-20] in the EMERGE patients was significantly higher than in the RELIEFE patients: 16 [12-20] ( $p<0.001$; data not shown). Interestingly, RELIEF patients had significantly higher scores for some symptoms, including lump sensation, cough, dyspnea, hoarseness, and throat clearing, whereas EMERGE patients had higher scores for heartburn and difficult swallowing. So, the clinical features were really different in the two populations, such as GERD and LPR patients.

Considering the previous treatments, the frequency of past/current treatments in the EMERGE group (Figure 2A) was significantly higher than in RELIEF group (Figure 2 B) patients $(p<0.0001$ ): this outcome underlines the more intense recourse to medical therapy in GERD patients than in LPR patients. Analyzing the treatment options, Figure 3 shows the distribution of different types of treatments prescribed in the past (panel A, C) or currently used (panel B, D): monotherapy with PPI, PPI in add-on, and Miscellany in EMERGE (panel A, B) and RELIEF (panel C, D) patients. LPR patients were more frequently treated with PPI as monotherapy and with miscellany treatments, whereas GERD patients use more commonly PPI plus add-on, even though PPI alone are the first-choice therapy also in GERD. Considering the distribution of the new prescribed

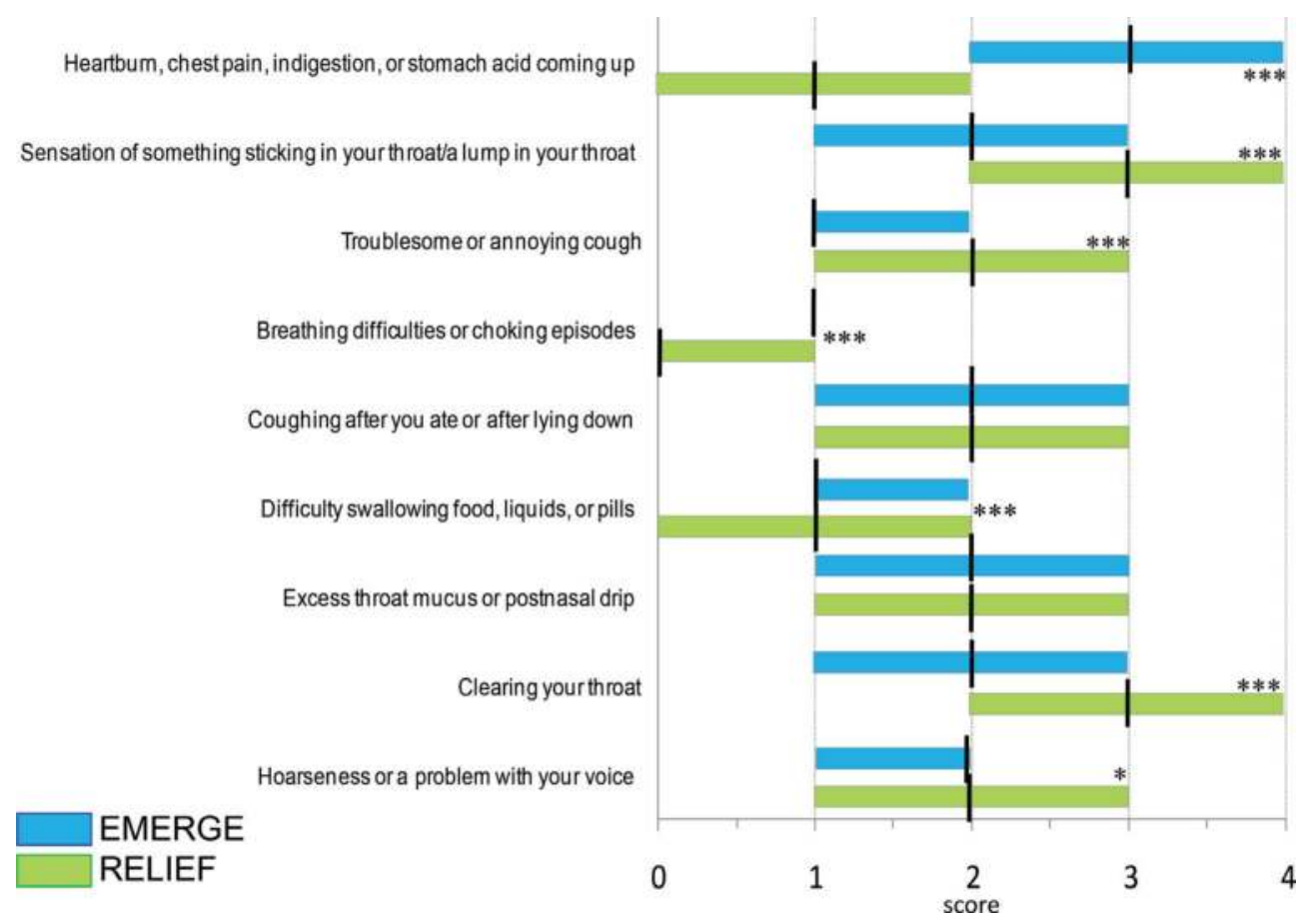

Figure 1. Reflux Symptom Index (RSI) as median values for each single symptom in the EMERGE (no. 789) and in the RELIEF population (no. 1934). Scores are reported as medians (bars) with lower and upper quartiles (boxes). 


\section{A. EMERGE (No. 754)}

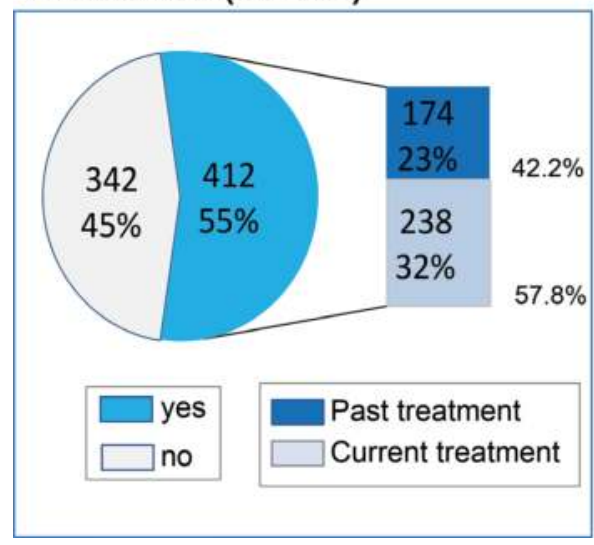

B. RELIEF (No. 1,685)

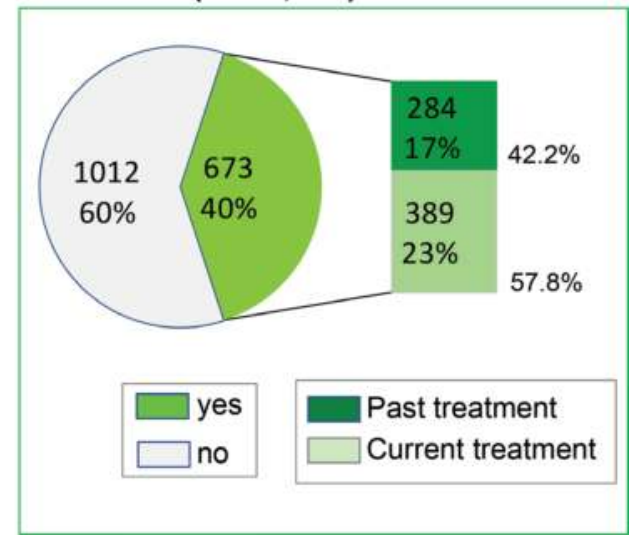

Figure 2. Distribution of the EMERGE (panel A) or RELIEF (panel B) patients according to the treatments: past, current, or never.
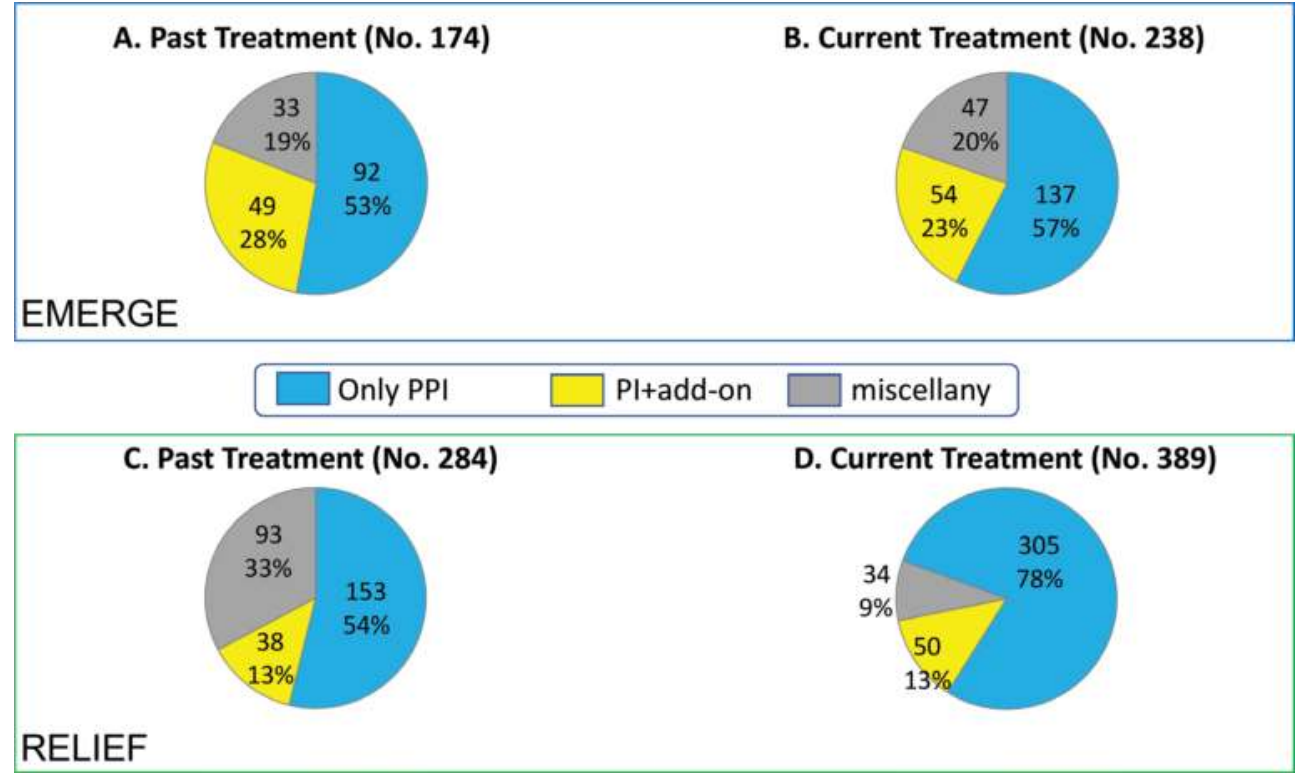

Figure 3. Distribution of different types of treatments prescribed in the past (panel A, C) or currently used (panel B, D): monotherapy with PPI, PPI in add-on, and miscellany in EMERGE (panel A, B) and RELIEF (panel C, D) patients.

treatments (i.e., prescriptions made during the visits) and specifically of Marial® as monotherapy, PPI as monotherapy or PPI in add-on, there was difference in EMERGE (Figure 4A) and in RELIEF (Figure 4B) patients $(\mathrm{p}<0.0001)$ : LPR patients were preferentially treated with Marial ${ }^{\circledR}$ alone, whereas GERD patients were treated essentially with PPI plus add-on. 


\section{A. EMERGE (No. 755)}

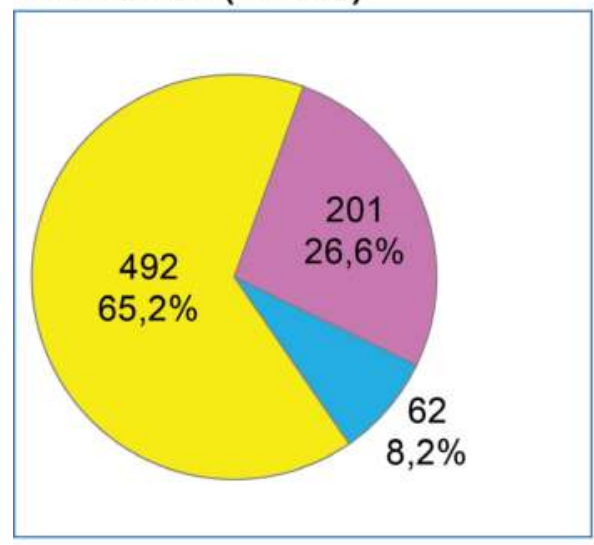

\section{B. RELIEF (No. 1,830)}

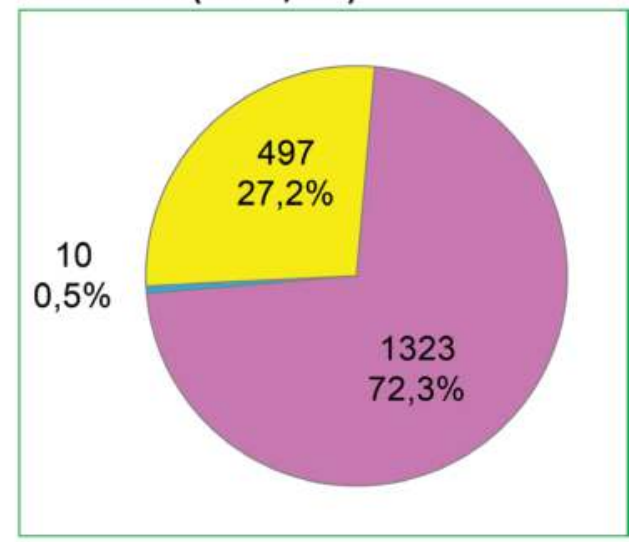

Only PPI

\section{Pl+add-on}

miscellany

Figure 4. Distribution of the Marial ${ }^{\circledR}$ as monotherapy, PPI as monotherapy or PPI in add-on in new prescribed treatments (i.e., prescription during the visit) in EMERGE (panel A) and RELIEF (panel B) patients.

Heartburn, chest pain, indigestion, or stomach acid coming up

Sensation of something sticking in the throat or a lump in the throat

Troublesome or annoying cough

Breathing difficulties or choking episodes

Coughing after having eaten or after lying down

Difficulty swallowing food, liquids, or pills

Excess throat mucus or postnasal drip

Clearing the throat

Hoarseness/problem with the voice

\section{EMERGE} RELIEF

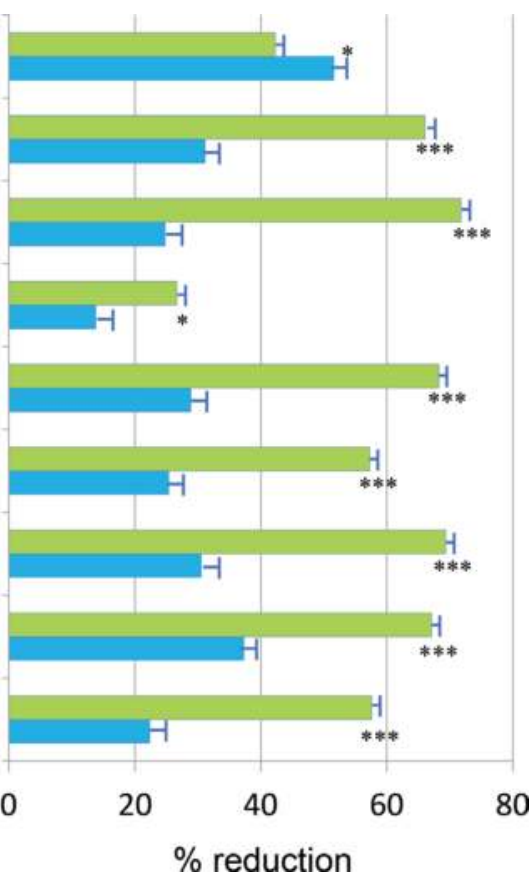

Figure 5. Reduction in RSI values for each single symptom before and after a 4 week-treatment with Marial ${ }^{\circ}$ as monotherapy in EMERGE and in RELIEF patients. 
Comparing the patients' perception of treatment efficacy, reduction in RSI values for each single symptom before and after a 4 week-treatment with Marial ${ }^{\circledR}$ alone or with PPI in addon in EMERGE and RELIEF patients are reported in Figures 5 and 6. Marial ${ }^{\circledR}$ alone treatment induced a statistically significant higher reduction in each single symptom in RELIEF patients than in EMERGE patients, with the exception of heartburn, chest pain, indigestion, or stomach acid coming up (Figure 5). Similar results were obtained evaluating the reduction in RSI values in patients treated with PPI in add-on that was able to determine a higher statistically significant decrease in RELIEF than in EMERGE patients in each single symptom, with the exception of heartburn, chest pain, indigestion, or stomach acid coming up (Figure 6).

In conclusion, these two surveys provided some interesting outcomes: (i) diagnostic questionnaires (RSI, RFS, and GIS) are reliable and useful both during the visit and to orient the treatment decision; (ii) GERD and LPR present different clinical features; (iii) as consequence the treatments (both previous and actual) are different; (iv) the introduction of Marial ${ }^{\circledR}$ significantly affected the otolaryngologist approach and partially the gastroenterologist orientation; (v) Marial ${ }^{\circledR}$ was effective both in LPR and GERD patients; (vi) Marial ${ }^{\circledR}$ showed more effectiveness than conventional therapy (PPI plus add-on); and (vii) LPR patients were more responsive to medical treatments than GERD patients. Therefore, these outcomes may give a pragmatic usefulness to both otolaryngologists and gastroenterologists in clinical practice.

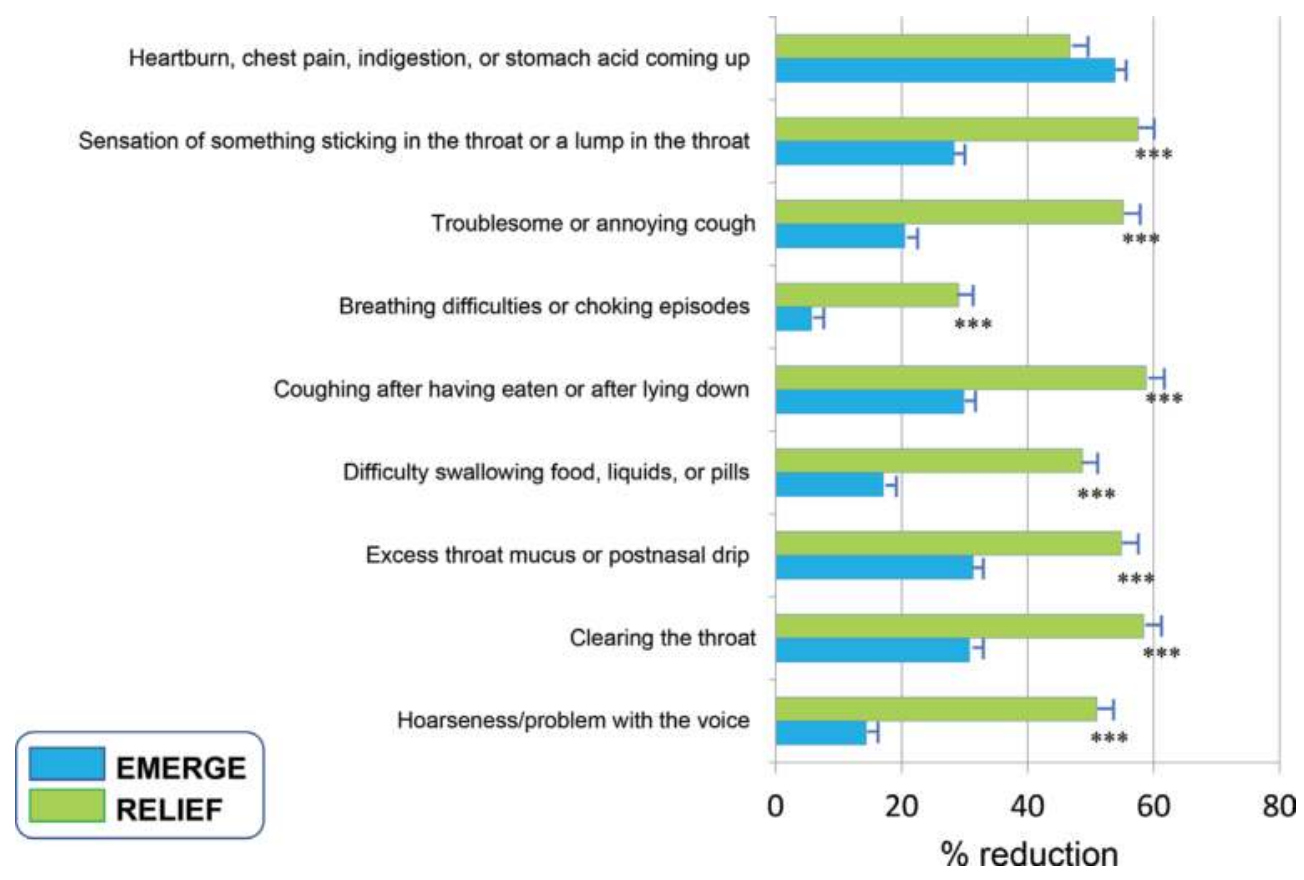

Figure 6. Reduction in RSI values for each single symptom before and after a 4 week-treatment with PPI + add-on in EMERGE and in RELIEF patients. 


\section{Author details}

Aragona Salvatore Emanuele ${ }^{1}$, Mereghetti Giada ${ }^{1}$ and Giorgio Ciprandi ${ }^{2 *}$

*Address all correspondence to: gio.cip@libero.it

1 Center of Regenerative Medicine, Humanitas Mater Domini, Castellanza (VA), Italy

2 Ospedale Policlinico San Martino, Genoa, Italy

\section{References}

[1] Sidhwa F, Moore A, Alligood E, Fisichella PM. Diagnosis and treatment of the extraesophageal manifestations of gastroesophageal reflux disease. Annals of Surgery. 2017; 265:63-67

[2] Rubenstein JH, Chen JW. Epidemiology of gastroesophageal reflux disease. Gastroenterology Clinics of North America. 2014;43:1-14

[3] Francis DO, Rymer JA, Slaughter JC, et al. High economic burden of caring for patients with suspected extraesophageal reflux. The American Journal of Gastroenterology. 2013;108:905-911

[4] Schappert SM, Burt CW. Ambulatory care visits to physician offices, hospital outpatient departments, and emergency departments: United States, 2001-02. Vital and Health Statistics. 2006:1-66

[5] Vakil N, van Zanten SV, Kahrilas P, Dent J, Jones R, Global Consensus Group. The Montreal definition and classification of gastroesophageal reflux disease: A global evidence-based consensus. The American Journal of Gastroenterology. 2006;101:1900-1920

[6] Katz P, Gerson LB. Corrigendum: Guidelines for the diagnosis and management of the gastroesophageal reflux disease. The American Journal of Gastroenterology. 2013;108: 308-328

[7] Savarino E. NERD: An umbrella term including heterogenous subpopulations. Nature Reviews. Gastroenterology \& Hepatology. 2013;10:371-380

[8] Quasim A. Section II FGIDs: Diagnostic groups. Esophageal disorders. Gastroenterology. 2016;150:1368-1379

[9] Salihefendic N, Zidzic M, Cabric E. Laryngopharyngeal reflux disease-LPRD. Medical Archives. 2017;71:215-218

[10] Capagnolo AM, Priston J, Heidrichthoen R, Medeiros T, Assuncao RA. Laryngopharyngeal reflux: Diagnosis, treatment and latest research. International Archives of Otorhinolaryngology. 2014;18:184-191 
[11] Dent J, El-Serag HB, Wallander MA, Johansson S. Epidemiology of gastroesophageal reflux disease: A systematic review. Gut. 2005;54:710-717

[12] Koufman JA. Laryngopharyngeal reflux 2002: A new paradigm of airway disease. Ear, Nose, \& Throat Journal 2004; (Suppl. 1):10-29

[13] Saritas Yuksel E, Vaezi MF. New developments in extraesophageal reflux disease. Gastroenterología y Hepatología. 2012;8:590-599

[14] Barry DW, Vaezi MF. Laryngopharyngeal reflux: More questions than answers. Cleveland Clinic Journal of Medicine. 2010;77:327-334

[15] Belafsky PC, Postma GN, Amin MR, Koufman JA. Symptoms and findings of laryngopharyngeal reflux. Ear, Nose, \& Throat Journal 2004; (Suppl. 1):30-39

[16] Abou-Ismail A, Vaezi MF. Evaluation of patients withsuspected laryngopharyngeal reflux: A practical approach. Current Gastroenterology Reports. 2011;13:213-218

[17] Martinucci I, de Bortoli N, Savarino E, Nacci A, Romeo SO, Bellini M, Savarino V, Fattori B, Marchi S. Optimal treatment of laryngopharyngeal reflux disease. Therapeutic Advances in Chronic Disease. 2013;4:287-301

[18] Kung YM, Hsu WH, Wu MC, Wang JW, Liu CJ, Su YC, et al. Recent advances in the pharmacological management of gastroesophageal reflux disease. Digestive Diseases and Sciences. 2017;62:3298-3316

[19] Sigterman KE, van Pinxteren B, Bonis PA, Lau J, Numans ME. Short-term treatment with proton pump inhibitors, H2-receptor antagonists and prokinetics for gastroesophageal reflux disease-like symptoms and endoscopy negative reflux disease. Cochrane Database of Systematic Reviews. 2013:CD002095

[20] Sifrim D, Zerbib F. Diagnosis and management of patients with reflux symptoms refractory to proton pump inhibitors. Gut. 2012;61:1340-1354

[21] Fass R, Shapiro M, Dekel R, Sewell J. Systematic review: Proton-pump inhibitor failure in gastro-oesophageal reflux disease-Where next? Alimentary Pharmacology \& Therapeutics. 2005;22:79-94

[22] Fass R. Proton-pump inhibitor therapy in patients with gastrooesophageal reflux disease: Putative mechanisms of failure. Drugs. 2007;67:1521-1530

[23] Fass R, Gasiorowska A. Refractory GERD: What is it? Current Gastroenterology Reports. 2008;10:252-257

[24] Scarpellini E, Ang D, Pauwels A, et al. Management of refractory typical GERD symptoms. Nature Reviews. Gastroenterology \& Hepatology. 2016;13:281-294

[25] Kahrilas PJ, McColl K, Fox M, et al. The acid pocket: A target for treatment in reflux disease? The American Journal of Gastroenterology. 2013;108:1058-1064

[26] Mitchell DR, Derakhshan MH, Robertson EV, McColl KE. The role of the acid pocket in gastroesophageal reflux disease. Journal of Clinical Gastroenterology. 2016;50:111-119 
[27] Strugala V, Avis J, Jolliffe IG, Johnstone LM, Dettmar PW. The role of an alginate suspension on pepsin and bile acids-key aggressors in the gastric refluxate. Does this have implications for the treatment of gastro-oesophageal reflux disease? The Journal of Pharmacy and Pharmacology. 2009;61:1021-1028

[28] Kwiatek MA, Roman S, Fareeduddin A, Pandolfino JE, Kahrilas PJ. An alginate-antacid formulation (Gaviscon double action liquid) can eliminate or displace the postprandial 'acid pocket' in symptomatic GERD patients. Alimentary Pharmacology \& Therapeutics. 2011;34:59-66

[29] Rohof WO, Bennink RJ, Smout AJ, Thomas E, Boeckxstaens GE. An alginate-antacid formulation localizes to the acid pocket to reduce acid reflux in patients with gastroesophageal reflux disease. Clinical Gastroenterology and Hepatology. 2013;11:1585-1591

[30] Sweis R, Kaufman E, Anggiansah A, et al. Post-prandial reflux suppression by a raftforming alginate (Gaviscon advance) compared to a simple antacid documented by magnetic resonance imaging and $\mathrm{pH}$-impedance monitoring: Mechanistic assessment in healthy volunteers and randomised, controlled, double-blind study in reflux patients. Alimentary Pharmacology \& Therapeutics. 2013;37:1093-1102

[31] Dettmar PW, Sykes J, Little SL, Bryan J. Rapid onset of effect of sodium alginate on gastro-oesophageal reflux compared with ranitidine and omeprazole, and relationship between symptoms and reflux episodes. International Journal of Clinical Practice. 2006;60:275-283

[32] De Ruigh A, Roman S, Chen J, Pandolfino JE, Kahrilas PJ. Gaviscon double action liquid (antacid \& alginate) is more effective than antacid in controlling post-prandial oesophageal acid exposure in GERD patients: A double-blind crossover study. Alimentary Pharmacology \& Therapeutics. 2014;40:531-537

[33] Mandel KG, Daggy BP, Brodie DA, Jacoby HI. Review article: Alginate-raft formulations in the treatment of heartburn and acid reflux. Alimentary Pharmacology \& Therapeutics. 2000;14:669-690

[34] Lai IR, Wu MS, Lin JT. Prospective, randomized, and active controlled study of the efficacy of alginic acid and antacid in the treatment of patients with endoscopy-negative reflux disease. World Journal of Gastroenterology. 2006;12:747-754

[35] Pouchain D, Bigard MA, Liard F, et al. Gaviscon(R) vs. omeprazole in symptomatic treatment of moderate gastroesophageal reflux. A direct comparative randomised trial. BMC Gastroenterology. 2012;12:18

[36] Woodland P, Lee C, Duraisamy Y, et al. Assessment and protection of esophageal mucosal integrity in patients with heartburn without esophagitis. The American Journal of Gastroenterology. 2013;108:535-543

[37] Sun J, Yang C, Zhao H, et al. Randomised clinical trial: The clinical efficacy and safety of an alginate-antacid (Gaviscon double action) versus placebo, for decreasing upper gastrointestinal symptoms in symptomatic gastroesophageal reflux disease (GERD) in China. Alimentary Pharmacology \& Therapeutics. 2015;42:845-854 
[38] Chiu CT, Hsu CM, Wang CC, et al. Randomised clinical trial: Sodium alginate oral suspension is non-inferior to omeprazole in the treatment of patients with non-erosive gastroesophageal disease. Alimentary Pharmacology \& Therapeutics. 2013;38:1054-1064

[39] Manabe N, Haruma K, Ito M, et al. Efficacy of adding sodium alginate to omeprazole in patients with nonerosive reflux disease: A randomized clinical trial. Diseases of the Esophagus. 2012;25:373-380

[40] Glicksman JT, Mick PT, Fung K, Carroll TL. Prokinetic agents and laryngopharyngeal reflux disease: A systematic review. Laryngoscope. 2014;124:2375-2379

[41] McGlashan JA, Johnstone LM, Sykes J, Strugala V, Dettmar PW. The value of a liquid alginate suspension (Gaviscon advance) in the management of laryngopharyngeal reflux. European Archives of Oto-Rhino-Laryngology. 2009;266:243-251

[42] Zerbib F, Dulery C. Facts and fantasies on extraesophageal reflux. A gastroenterologist perspective. Journal of Clinical Gastroenterology. 2017;51:769-776

[43] Vertigan AE, Bone SL, Gibson PG. Laryngeal sensory dysfunction in laryngeal hypersensitivity syndrome. Respirology. 2013;18:948-956

[44] Aziz Q, Fass R, Gyawali CP. Functional esophageal disorders. Gastroenterology. In press. DOI: 10.1053/j.gastro.2016.02.012. [Epub ahead of print]

[45] Miller TA, Jacobson ED. Gastrointestinal cytoprotection by prostaglandins. Gut. 1979; 20:75-87

[46] Johansson C, Bergström S. Prostaglandin and protection of the gastroduodenal mucosa. Scandinavian Journal of Gastroenterology. 1982;77:21-46

[47] Tasman-Jones C. Pathogenesis of peptic ulcer disease and gastritis: Importance of aggressive and cytoprotective factors. Scandinavian Journal of Gastroenterology. 1986: 122:1-122:5

[48] Orlando RC. Cytoprotection by sucralfate in acid-exposed esophagus: A review. Scandinavian Journal of Gastroenterology. 1987;127:97-100

[49] Lauritsen K, Rask-Madsen J. Peptic ulcer disease, cytoprotection, and prostaglandins. Archives of Internal Medicine. 1990;150:695-701

[50] Chater PI, Wilcox MD, Brownlee IA. Alginate as a protease inhibitor in vitro and in a model gut system: Selective inhibition of pepsin but not trypsin. Carbohydrate Polymers. 2015;131:142-151

[51] Internal Clinical Guidelines Team (UK). Dyspepsia and Gastro-Oesophageal Reflux Disease: Investigation and Management of Dyspepsia, Symptoms Suggestive of GastroOesophageal Reflux Disease, or Both. London: National Institute for Health and Care Excellence (UK); 2014 Sep

[52] Davies I, Burman-Roy S, Murphy MS, Guideline Development Group. Gastro-oesophageal reflux disease in children: NICE guidance. BMJ. 2015;350:7703 
[53] Aragona SE, Mereghetti G, Ciprandi G. The gastric reflux: the therapeutical role of Marial. Journal of Biological Regulators and Homeostatic Agents. 2018 Apr 26;32(4). [Epub ahead of print]

[54] Bianchetti M, Peralta S, Nicita R, Aragona SE, Ciprandi G. The EMERGE (emerging from Gastroesophageal Reflux): an Italian survey-I. The viewpoint of the Gastroenterologist. Journal of Biological Regulators and Homeostatic Agents. 2018 Apr 26;32(4). [Epub ahead of print]

[55] Bianchetti M, Peralta S, Nicita R, Aragona SE, Ciprandi G. The EMERGE (emerging from Gastroesophageal Reflux): an Italian survey-II. The viewpoint of the patient. Journal of Biological Regulators and Homeostatic Agents. 2018 Apr 26;32(4). [Epub ahead of print]

[56] Bianchetti M, Peralta S, Nicita R, Aragona SE, Ciprandi G. Correlation between the Reflux Finding Score and the GERD Impact Scale in patients with Gastroesophageal Reflux Disease. Journal of Biological Regulators and Homeostatic Agents. 2018 Apr 26;32(4). [Epub ahead of print] 
